\title{
Stochastic Dynamics of Anæsthesia
}

\author{
A. Bandrivskyy*, M. Entwistle ${ }^{\dagger}$, P.V.E. McClintock*, B. Musizza*, ${ }^{*}$,

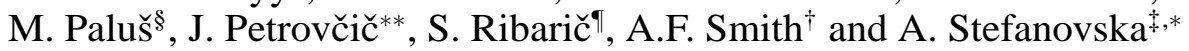 \\ *Department of Physics, Lancaster University, Lancaster LA1 4YB, UK \\ ${ }^{\dagger}$ Department of Ancesthesia, Royal Lancaster Infirmary, Ashton Road, Lancaster LA1 4RP, UK \\ ** Jožef Stefan Institute, Jamova 39, Ljubljana, Slovenia \\ $¥$ Group of Nonlinear Dynamics and Synergetics, Faculty of Electrical Engineering, University of \\ Ljubljana, Tržaška 25, Ljubljana, Slovenia \\ ${ }^{\S}$ Institute of Computer Science, Academy of Sciences of the Czech Republic, \\ Pod vodárenskou věží 2, 18207 Prague 8, Czech Republic \\ II Institute of Pathophysiology, Faculty of Medicine, University of Ljubljana, Zaloška 7, Ljubljana,
} Slovenia

\begin{abstract}
.
Recent developments in the analysis of synchronization and directionality of couplings for noisy nonlinear oscillators are being applied to study the complex interactions between cardiac and respiratory oscillations, and brain waves (especially delta and gamma), during anæsthesia. It is found that marked changes occur in the inter-oscillator interactions during anæsthesia in both rats and humans. These could form a new basis for measurement of depth of anæsthesia. The new EC programme BRACCIA will explore and quantify causal relationships between the oscillatory processes for the different stages of anæsthesia and consciousness.
\end{abstract}

Keywords: Synchronization, noise, nonlinear oscillator, directionality, anæsthesia, cardiovascular system, phase transition

\section{INTRODUCTION}

There is abundant evidence favouring the perception of the cardiovacular system as a noisy dynamical system that behaves much like a collection of coupled oscillators [1]. The inter-oscillator couplings give rise to many of the complex and emergent phenomena seen in classical oscillator systems, including chaos [2,3], modulation [1] and episodes of synchronization [4, 5, 6]. Numerical modelling of the cardiovascular system [7] has shown that the role of noise in facilitating these phenomena can be crucial. Yet the physiological origin of the oscillators is not yet fully established, nor the nature of the interactions between them, nor the origins or character of the the noise itself. The nonlinear dynamics of anæsthesia therefore represents par excellence an unsolved problem of noise.

It has become clear that the oscillatory processes - their amplitudes, frequencies and especially their mutual couplings - can be used to characterise the state of the system. It is known, for example, that that marked changes in cardio-respiratory synchronisation occur in anæsthesia [8]; furthermore, recent work by two of us [9] has shown that cerebral (cortical) oscillations interact with those of the cardiac and respiratory systems and that these interactions change in anæsthesia. Although the work is in its infancy, and is extremely challenging, there is a clear opportunity for applying techniques from 
stochastic nonlinear dynamics, information theory and control theory to illuminate the underlying physiological processes.

Application of the techniques of nonlinear science to rhythmic signals of biological origin [10] has already illuminated many fundamental processes, especially those connected with cardiac function. These include fractal dynamics [11], deterministic chaos [3] in heart rate variability (HRV) data and the fact (see above) that the blood circulatory system behaves in many respects like a collection of five coupled oscillators [1] with frequencies ranging from $1 \mathrm{~Hz}$ (cardiac) down to $0.01 \mathrm{~Hz}$ (endothelial/metabolic [12]). Episodes of cardio-respiritory synchronization are known [4, 8] to occur in humans; synchronization phenomena involving a $0.1 \mathrm{~Hz}$ blood flow component, believed to be of myogenic origin, can also occur [13, 14]. Pomfrett suggested [15] that HRV or a bispectral index could be used to quantify depth of anæsthesia but that, in practice, it will probably be better to use several indicators rather than to rely on any particular technology; Larson and Galletly have commented [16] that the standard methods used for HRV analysis are limited in their ability to detect the presence of cardioventilatory coupling in heart rate time series, although there are some graphical methods $[17,18]$ that seem promising. A different approach, based on synchronization indices and synchrograms, has recently been used to reveal an unexpected and very marked increase of cardio-respiratory synchronization during anæsthesia [8] in rats.

\section{ANESTHESIA}

In anæsthesia a chemical perturbation of the organism leads to a temporary loss of consciousness, but just how this happens remains to a large extent a mystery. Perhaps on account of this lack of deep understanding, there are not yet any reliable markers for depth of anæsthesia. Consequently, as Pomfrett [15] puts it -

“...the anæsthetist is still unable to measure the depth of anæsthesia in order to prevent inadvertent awakening during anæsthesia."

It is perhaps not surprising, therefore, that the incidence of awareness among patients undergoing surgery is nonzero: see Table 1 . Note that even the lowest estimate of $0.2 \%$ is unacceptably high. The problem can be particularly severe in cases where for clinical reasons the anæsthesia is kept as light as possible (e.g. Cæsarian section), and where a mechanically ventilated curarised patient is unable to give any voluntary indication of awareness.

A well advertised approach to assessment of depth of anæsthesia is the bispectral index technique known as BIS [20]. Its use is controversial, however, and it is certainly not $100 \%$ accurate [21, 19]. Other promising approaches involves measurements of changes in heart rate variability (HRV) [15] and cardio-respiratory coupling [8, 17]. What we propose in this project takes such ideas much further, partly by adopting a more sophisticated mathematical analysis of the data, and partly by including consideration of the neural (cortical) oscillations. 
TABLE 1. Incidence of awareness during surgery (after Pomfrett [15] and Myles at al [19]).

\begin{tabular}{|l|ccc|}
\hline Author & Date & Sample & Awareness \% \\
\hline Hutchinson & 1960 & 656 & 1.2 \\
Harris & 1971 & 120 & 1.6 \\
McKenna & 1973 & 200 & 1.5 \\
Wilson & 1975 & 490 & 0.8 \\
Liu et al & 1990 & 1000 & 0.2 \\
Myles et al & 2004 & 1238 & 0.9 \\
\hline
\end{tabular}
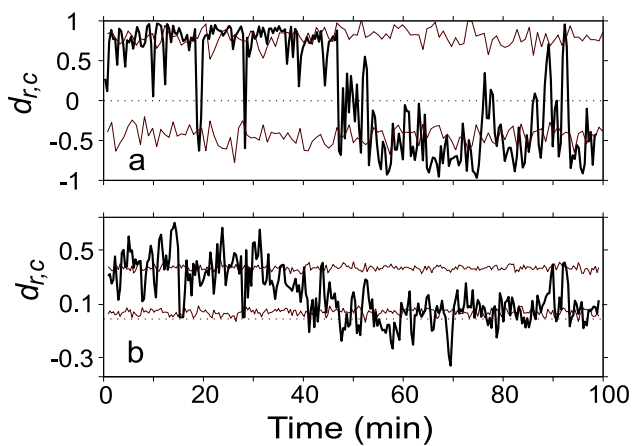

FIGURE 1. Direction of the cardio-respiratory interaction measured for a rat during anæsthesia. Top: calculated from the data according to the method proposed by Rosenblum et al [22]. Bottom: calculated from the same data using mutual information as proposed by Paluš et al [23]. The nearly horizontal thin lines are plotted \pm 2 standard deviations outside a set of surrogate data. If $d_{r, c}>0$ it means that respiration drives cardiac function, and vice versa if $d_{r, c}<0$. In each case the directionality index exhibits a change of sign at a time that coincides approximately with the end of deep anæsthesia ([24]).

\section{Anæsthesia in rats}

The first simultaneous studies of the neural and cardio-respiratory phenomena associated with anæsthesia were carried out on rats, revealing a number of interesting effects. Rats exhibit similar cardiovascular dynamics to humans, except that the oscillatory components are 3-4 times faster [25], with heart beat at $\sim 3 \mathrm{~Hz}$ and respiration at $\sim 1 \mathrm{~Hz}$. Analyses based on techniques drawn from nonlinear science [22] and information theory $[23,26]$ are being used to reveal and quantify causal relationships between the cardiac, respiratory and neural oscillations, and how these change during anæsthesia. Several important results have emerged, and show that the more systematic study now proposed is likely to yield important results. As one example, we show in Fig. 1 how the cardio-respiratory directionality index evolves as deep anæsthesia gives way to lighter anæsthesia. There is a dramatic change of sign showing that, during the lighter anæsthesia, the heart is predominantly driving respiration rather than vice versa as is observed in the conscious state or in deep anæsthesia.

Computation of the cardio-respiratory directionality index and how it evolves with 

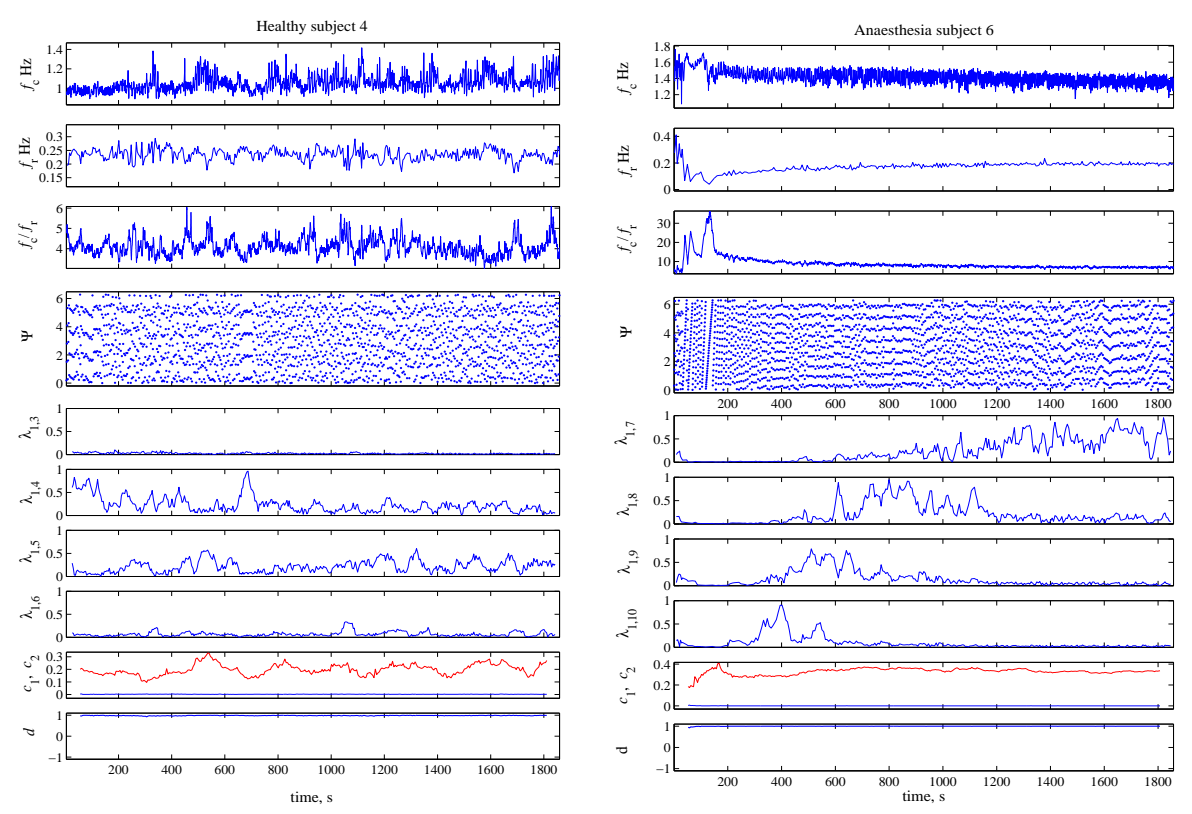

FIGURE 2. Typical analysis of cardiorespiratory data from human volunteers in the wakeful state (left) and under anæsthesia (right). From the top in each case, the plots show as functions of time: cardiac $f_{c}$ and respiratory $f_{r}$ frequencies and their ratio $f_{r} / f_{c}$; synchrogramme $\Psi$; synchronization indices $\lambda_{1, n}$; coupling coefficients $c_{1}$ and $c_{2}$; and directionality index $d$.

time from measured data could therefore provide one way of assessing depth of anæsthesia. There are several others: from the original research [8] it appears that strength of synchronization, synchronization index, and respiratory rate variability are good indicators; the latest results show that, in addition to the cardio-respiratory directionality index (Fig. 1), characteristic changes occur in the brain, especially $\delta$ and $\gamma$ waves, and in the interactions and causalities with the cardiac and respiratory oscillations.

An initial modelling of coupled oscillators within both the micro and macroscopic worlds and has been undertaken [27]. A large number of oscillators acting on a similar time scale has been be represented as an ensemble that, on the macroscopic scale, may be taken as a single oscillator. Furthermore, their interaction on the macroscopic scale with other similar type of oscillators has been described, but acting on widely different time scales.

\section{Anæsthesia in humans}

The crucially important question of "Does what applies to rats also apply to humans?", i.e. whether anæsthetised humans display similar phenomena to those seen earlier in rats, is being addressed. The preliminary investigation [28] has involved studies of ten healthy 
men aged between 20 and 40 undergoing elective surgery. Standard clinical monitoring was supplemented by forehead BIS Quatro electrodes (Aspect Medical Systems, Leiden) for EEG capture and a TSD201 respiratory effort transducer (BIOPAC Systems Inc, Goleta, CA) for respiratory frequency (f). A standard anæsthetic (midazolam $2 \mathrm{mg}$, fentanyl $1.5 \mu \mathrm{gkg}^{-1}$, sleep-dose propofol and isoflurane $1 \%$ in $40 \%$ oxygen in air) was administered by the same anæsthetist. Recordings were made for 30 minutes in the anæsthetic room prior to surgery. Waveforms were digitised at $1000 \mathrm{~Hz}$ and stored on a $\mathrm{PC}$ for later analysis.

A typical set of results is shown in Fig. 2 for healthy subjects in the waking (left) and anæsthetised (right) states. The synchrogramme $\Psi$ plots points of fixed phase of the cardiac oscillator as a function of the phase of the respiratory oscillator, and therefore yields $n$ horizontal lines where there is $1: n$ synchronisation; the synchronisation index $\lambda_{1, n}$, calculated from the data, is equal to unity for perfect $1: n$ synchronisation, but is zero in its absence; the coupling coefficients $c_{1}, c_{2}$ provide measures of the cardiac-to-respiratory (lower lines) and respiratory-to-cardiac (upper lines) couplings; and the directionality index $d$ specifies the direction of coupling, being +1 for exclusively respiratory-tocardiac coupling, and -1 for the opposite extreme. In all cases the idealised behaviour is to some extent modified by noise. In the waking state, the synchrogramme reveals two brief episodes of 1:4 synchronisation starting at 0 and $650 \mathrm{~s}$ into the measurements. Under anæsthesia, in marked contrast, there is a virtually continuous state of synchronization as shown by the horizontal lines in the synchrogramme and finite values of the synchronization indices. As time evolves, the system passes through 1:10, 1:9, 1:8 and 1:7 synchronized states - very similar to the behaviour seen earlier in rats [8]. The directionality index $d$, calculated from the two unidirectional coupling coefficients $c_{1}, c_{2}$, shows that during deep anæsthesia, as in the waking state, respiration tends to drive the cardiac oscillator. In rats, $d$ changes sign during emergence from anæsthesia (Fig. 1); it is not yet known what happens for humans.

We now plan to monitor patients throughout surgery and into recovery to explore how synchronisation and directionality alter with changing depth of anaesthesia.

\section{CONCLUSION}

Many problems remain to be solved. In particular it will be necessary to develop algorithms for calculation of the directionality indices that are robust in the presence of physiological noise. It is also highly desirable to reach an understanding of the underlying processes giving rise to interactions between the oscillatory processes. If such problems can be solved, the potential impact of the work is high, in both specific and general terms. Specifically in relation to anæsthesia, we note that: anæsthesia is universally employed throughout the world for all but the simplest surgery; awareness during anæsthesia is not as uncommon as it should be (Table 1) and, as already mentioned, a better way of measuring depth of anæsthesia would be widely welcomed. More generally, we note that: coupled oscillators are widespread in nature, at all levels; and causal relationships between such oscillators are of great interest because they shed light on the underlying mechanisms of coupling. Improved techniques for establishing causalities based on measured noisy time series will be applicable in many areas. 


\section{REFERENCES}

1. A. Stefanovska, and M. Bračič, Contemporary Phys., 40, 31-55 (1999).

2. A. Babloyantz, and A. Destexhe, Biol. Cybern., 58, 203-211 (1988).

3. C. S. Poon, and C. K. Merrill, Nature, 389, 492-495 (1997).

4. C. Schäfer, M. G. Rosenblum, J. Kurths, and H. H. Abel, Nature, 392, 239-240 (1998).

5. C. Schäfer, M. G. Rosenblum, H. H. Abel, and J. Kurths, Phys. Rev. E, 60, 857-870 (1999).

6. A. Pikovsky, M. Rosenblum, and J. Kurths, Synchronization - A Universal Concept in Nonlinear Sciences, Cambridge University Press, Cambridge, 2001.

7. A. Stefanovska, D. G. Luchinsky, and P. V. E. McClintock, Physiol. Meas., 22, 551-564 (2001).

8. A. Stefanovska, H. Haken, P. V. E. McClintock, M. Hožič, F. Bajrović, and S. Ribarič, Phys. Rev. Lett., 85, 4831-4834 (2000).

9. B. Musizza, and A. Stefanovska, "Interactions between cardiac, respiratory and brain activity in humans," in Fluctuations and Noise in Biological, Biophysical and Biomedical Systems III, edited by N. G. S. et al, SPIE, Bellingham, 2005, vol. 5841, pp. 139-149.

10. L. Glass, Nature, 410, 277-284 (2001).

11. A. L. Goldberger, L. A. N. Amaral, J. M. Hausdorff, P. C. Ivanov, C.-K. Peng, and H. E. Stanley, Proc. National Acad. of Sciences Suppl. 1, 99, 2466-2472 (2002).

12. H. D. Kvernmo, A. Stefanovska, K.-A. Kirkebøen, and K. Kvernebo, Microvasc. Res., 57, 298-309 (1999).

13. A. Stefanovska, and M. Hožič, Progress of Theoretical Physics Supplement, 139, 270-282 (2000).

14. N. B. Janson, A. G. Balanov, V. S. Anishchenko, and P. V. E. McClintock, Phys. Rev. E, 65, 036212 (2002).

15. C. J. D. Pomfrett, Brit. J. Ancesth., 82, 659-662 (1999).

16. P. D. Larson, and D. C. Galletly, Br. J. of Ancesth., 87, 819-826 (2001).

17. D. C. Galletly, and P. D. Larsen, Brit. J. Ancesth., 87, 827-833 (2001).

18. D. C. Galletly, and P. D. Larsen, Brit. J. Ancesth., 86, 777-788 (2001).

19. P. S. Myles, K. Leslie, J. McNeil, A. Forbes, and M. T. V. Chan, The Lancet, 363, 1757-1763 (2004).

20. J. M. Vernon, E. Lang, P. S. Sebel, and P. Manberg, Anesthesia and Analgesia, 80, 780-785 (1995).

21. C. Lennmarken, and R. Sandin, The Lancet, 363, 1747-1748 (2004).

22. M. G. Rosenblum, L. Cimponeriu, A. Bezerianos, A. Patzak, and R. Mrowka, Phys. Rev. E., 65, 041909 (2002).

23. M. Paluš, V. Komárek, Z. Hrnčíŕ, and K. Štěrbová, Phys. Rev. E, 63, 046211 (2001).

24. B. Musizza, A. Stefanovska, P. V. E. McClintock, M. Paluš, J. Petrovčič, and S. Ribarič, (in preparation) (2005).

25. F. Bajrović, M. Čenčur, M. Hožič, S. Ribarič, and A. Stefanovska, Pflüg. Arch.: Europ. J. Physiol. Supl., 439, R158-R160 (2000).

26. M. Paluš, and A. Stefanovska, Phys. Rev. E, 67, 055201(R) (2003).

27. B. Musizza, and A. Stefanovska, "Noise and synchronization on micro and macroscopic scales," in Fluctuations and Noise in Biological, Biophysical and Biomedical Systems II, edited by D. A. et al, SPIE, Bellingham, 2004, pp. 273-282.

28. M. Entwistle, A. Bandvivskyy, B. Musizza, A. Stefanovska, P. V. E. McClintock, and A. Smith, Br. J. of Anasthesia, 93, 608P-609P (2004). 
Copyright of AIP Conference Proceedings is the property of American Institute of Physics. The copyright in an individual article may be maintained by the author in certain cases. Content may not be copied or emailed to multiple sites or posted to a listserv without the copyright holder's express written permission. However, users may print, download, or email articles for individual use. 